\title{
Recurrent Generalized Seizures with Postictal Todd's Paralysis Caused by Medication-Associated Severe Hypomagnesemia: A Case Report
}

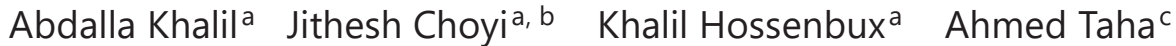 \\ ${ }^{a}$ Acute Medicine Department, King's College University Hospital Trust, Princess Royal \\ University Hospital, London, UK; ${ }^{b}$ Acute Stroke Department, King's College University \\ Hospital Trust, Princess Royal University Hospital, London, UK; 'Radiology Department, \\ King's College University Hospital Trust, Princess Royal University Hospital, London, UK
}

\section{Keywords}

Unprovoked first seizure $\cdot$ Hypomagnesemia-induced seizure $\cdot$ Todd's paralysis · Drugs associated with severe hypomagnesemia

\section{Abstract}

Hypomagnesemia is one of the electrolyte disturbances that can cause seizures. It is common in the hospitalized patients and can be induced by long-term usage of many medications. A 68-year-old male known to have hypertension and gastroesophageal reflux presented to the Emergency Department with an unprovoked first seizure at home followed by a temporary right-sided hemiparesis, dysphasia, and facial asymmetry. The hemiparesis, dysphasia, and facial asymmetry resolved within less than an hour after the seizure. His serum potassium was low with prolonged QT interval in the electrocardiogram (serum magnesium was not checked in the Emergency Department). He received intravenous IV potassium chloride infusion, and his serum potassium level was corrected, but he had a recurrent seizure after $10 \mathrm{~h}$. At that time, his serum magnesium was found to be very low, he received IV magnesium sulfate infusion, and his indapamide, omeprazole, and metformin medications were stopped. He had no further seizures, the rest of his blood tests were normal, and his CT brain was unremarkable. He was treated for aspiration pneumonia, and his outpatient MRI brain and EEG came to be normal too. 


\section{Introduction}

Electrolyte disturbances are commonly encountered in our daily clinical practice. Seizures are more frequently observed with hyponatremia, hypocalcemia, and hypomagnesemia [1]. Postictal temporary limb weakness (Todd's paresis/paralysis) that lasts a few minutes and up to $36 \mathrm{~h}$ is known to happen occasionally after either a focal (partial) or a generalized tonicclonic seizure [2]. There is a group of medications that are reported to cause hypomagnesemia. We are presenting a 68-year-old male who presented with recurrent seizures, postictal right hemiparesis, and dysphasia and was found to have severe hypomagnesemia caused by prescribed medications.

\section{Case Presentation}

A 68-year-old Caucasian male patient was escorted by an ambulance from home with a history of a sudden-onset slurred speech and right-sided upper limb weakness. His wife noted that the patient developed slurred speech and facial drooping, and then he became mute. She noted that the patient had jerky movements of the right arm, and then his whole body developed jerky movements before he developed the slurred speech. Paramedics could not assess the patient neurologically at home. The patient was confused and not obeying commands. His finger-prick blood glucose was $7.5 \mathrm{mmol} / \mathrm{L}$, and his observations were normal.

More detailed history could be taken in the ED after the patient regained his power, speech, and orientation. The patient did not have any history of vomiting, diarrhea, or fever. He had no previous history of seizures or stroke. He had a past medical history of diabetes mellitus type 2, essential hypertension, hypercholesterolemia, and ischemic heart disease and had 3 coronary stents in 2019.

His drug list includes aspirin, nicorandil, atorvastatin, indapamide, omeprazole, bisoprolol, and metformin. He denies any illicit drug intake and claimed that his alcohol intake is only recreational.

In the Emergency Department, his speech was normal, he was oriented, and his Glasgow Coma Scale was $15 / 15$. His power was $5 / 5$ bilaterally in both upper and lower limbs. His cranial nerves were normal, the plantar reflex was flexor, and no sensory deficit or cerebellar signs could be detected. The rest of the clinical examination was unremarkable.

His full blood count revealed a white blood count of $15.0 \times 109 / \mathrm{L}$ and neutrophils $9.0 \times$ 109/L. His renal profile was normal apart from potassium $2.7 \mathrm{mmol} / \mathrm{L}(3.5-5.0 \mathrm{mmol} / \mathrm{L})$. His liver function tests, serum calcium, phosphate, thyroid function tests, parathyroid hormone, and coagulation profile were normal.

His C-reactive protein was normal, venous blood gases were within normal levels, no metabolic alkalosis, lactic acid was $2.6 \mathrm{mmol} / \mathrm{L}$ (up to $2.0 \mathrm{mmol} / \mathrm{L}$ ), and glucose was $6.6 \mathrm{mmol} / \mathrm{L}$. CT brain was unremarkable. His ECG showed normal sinus rhythm with a heart rate of 75/min, prolonged QT interval $525 \mathrm{~ms}$ ( $<440 \mathrm{~ms}$ in men), and corrected QTc was $551 \mathrm{~ms}$ (Fig. 1).

While the patient was in the ED, he developed a second attack of generalized tonic-clonic seizures after momentary jerky movements of the right side of the body and received lorazepam IM. He regained consciousness and was able to move 4 limbs and speak freely after 30 min of right upper limb weakness, and no facial asymmetry was noted. He was started on IV potassium chloride $40 \mathrm{mmol} / \mathrm{L}$ infusion over $4 \mathrm{~h}$ and was admitted to the acute medical unit. In the ED, the Stroke team assessed the patient, and the impression was focal seizures with secondary generalization and postictal temporary aphasia and right hemiparesis. His evening repeated serum potassium was $3.5 \mathrm{mmol} / \mathrm{L}$.

\section{Karger'}




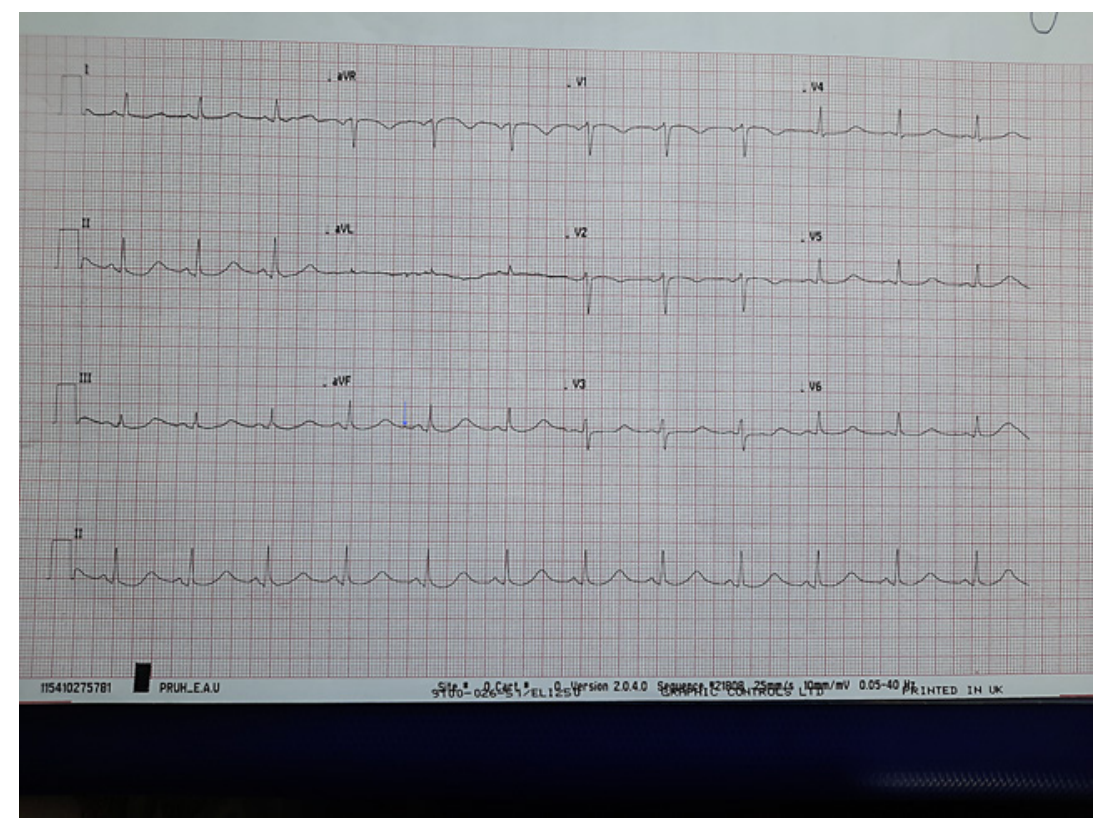

Fig. 1. Twelve-lead ECG showed normal sinus rhythm with a heart rate of $75 / \mathrm{min}$, prolonged QT interval $525 \mathrm{~ms}$ (<440 ms in men), and corrected QTc $551 \mathrm{~ms}$.

The day after admission, the patient developed a third attack of tonic-clonic seizure which lasted $60 \mathrm{~s}$. He was chesty, and oxygen saturation came down to 88\%.

He received a loading dose of IV levetiracetam, and blood was urgently taken for renal profile, bone profile, serum magnesium (which was not checked at ED the night before and not included within the bone profile), and venous blood gases. His chest examination revealed right basal crackles on chest auscultation.

The serum magnesium came to be $0.11 \mathrm{mmol} / \mathrm{L}(0.73-1.15 \mathrm{mmol} / \mathrm{L})$. He received an IV magnesium sulfate infusion and was transferred to an ECG monitored bed. His portable chest X-ray AP view showed right basal patchy shadowing suggestive of an infection (Fig. 2).

The WBC increased to $30.0 \times 109 / \mathrm{L}$, and his CRP became $150 \mathrm{mg} / \mathrm{L}(0-5 \mathrm{mg} / \mathrm{L})$. He was started on IV piperacillin/tazobactam for treating aspiration pneumonia in addition to levetiracetam $250 \mathrm{mg}$ twice daily orally.

The CT angiography of the brain was normal, and patient's serum magnesium normalized with IV replacement. He did not have any further seizures, and his oxygenation improved. Gradually he felt better, did not require any oxygen, and his white blood cells and CRP trended down to normal.

On the sixth day after admission, he was switched to oral antibiotics and was discharged home. He had an MRI brain as an outpatient which was normal, and his EEG revealed a normal background rhythm at $8 \mathrm{~Hz}$ and no definite epileptiform discharges.

\section{Discussion}

Hypomagnesemia is one of the electrolyte abnormalities that can cause seizures, and it is common in hospitalized patients (7-11\%) and is more frequent in critically ill patients and associated with higher mortality [3, 4]. Hypomagnesemia may be associated 
Fig. 2. Portable (mobile) chest X-ray revealed right basal patchy shadowing suggestive of infection.

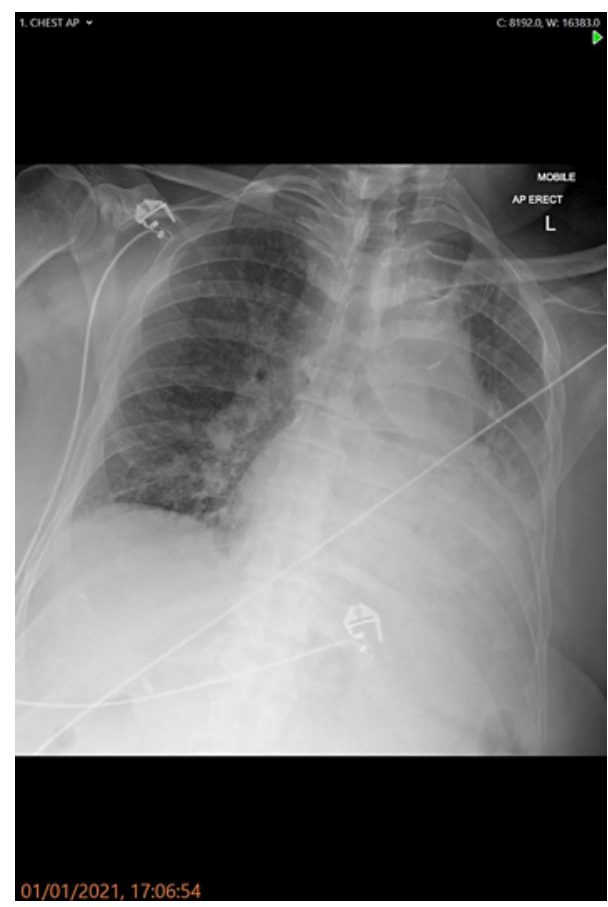

with other electrolyte disturbances mainly hypokalemia or hypocalcemia [5]. ECG changes of hypomagnesemia include prolonged QT interval, prolonged PR interval, $u$ wave, and atrial and ventricular ectopic, and it may cause ventricular tachycardia [6]. The prolonged QT interval and prominent $u$ wave are not specific for hypomagnesemia and maybe also noted with hypokalemia and carry a risk of torsade de pointes ventricular arrhythmia [7].

The American Academy of Neurology and the American Society of Epilepsy recommend checking blood glucose and serum electrolytes (sodium, potassium, calcium, and magnesium) for an adult patient presenting with unprovoked first seizures [8]. The incidence of Todd's paralysis following a seizure is $0.65-13 \%$ [9].

Our patient presented to the ED with a first seizure and without a history of trauma, drug withdrawal, or signs of meningeal irritation, and his CT head scan in ED was normal. His serum potassium was low, and his ECG showed prolonged QT interval. Initially, his serum magnesium was not checked in the ED. He was started on IV potassium chloride replacement which brought his potassium to $3.5 \mathrm{mmol} / \mathrm{L}$.

He developed a third seizure the day after admission, and his serum magnesium was found to be very low. He did not have any further seizures after IV magnesium replacement and stoppage of the incriminated medications.

Hypomagnesemia induced by long-term proton pump inhibitors has been reported and can recur if it was replaced with another proton pump inhibitor, but not with a histamine type-2 receptor antagonist such as famotidine $[10,11]$. The chronic usage of thiazides is also associated with hypomagnesemia and hypokalemia $[12,13]$.

Our patient was prescribed indapamide for essential hypertension and omeprazole for a suspected gastroesophageal reflux (both were prescribed for more than a year). Both drugs were canceled after the recurrence of seizures and the blood result showing severe hypomagnesemia. Metformin was also stopped because of the high lactic acid after recurrent seizures. The rest of the patient's blood tests, CT head, CT brain angiography, MRI brain, and EEG were unremarkable. 


\section{Conclusion}

Checking serum magnesium and other electrolytes is one of the required tests in the general workup of any adult patient presenting with an unprovoked first seizure. Hypomagnesemia may be associated with other electrolyte disturbances such as hypokalemia or hypocalcemia and may show similar ECG changes.

A thorough review of medications is essential for patients coming with first seizure and hypomagnesemia or other electrolyte disturbances. Immediate IV replacement of magnesium and transferring to a monitored bed will help to prevent recurrent unprovoked first seizures in adult patients with severe hypomagnesemia.

\section{Statement of Ethics}

This case report is exempt from ethics committee approval as per the UK National Service research governance arrangements. The authors have obtained written informed consent from the patient to publish his case report including images. All attempts have been made to make the case anonymous.

\section{Conflict of Interest Statement}

Authors declare no conflicts of interest with respect to publication or authorship of this case report.

\section{Funding Sources}

Authors declare not receiving any kind of funding from the public, commercial, or nonprofit sector.

\section{Author Contributions}

Dr. A.K. wrote the preliminary draft of the case report. Dr. C.J., Dr. K.H., and A.T. contributed and edited the final revision.

\section{Data Availability Statement}

All data related to this case report are available to be sent for review if requested, but not publically available.

\section{References}

1 Nardone R, Brigo F, Trinka E. Acute symptomatic seizures caused by electrolyte disturbances. J Clin Neurol. 2016 Jan;12(1):21-33.

2 Xu SY, Li ZX, Wu XW, Li L, Li CX. Frequency and pathophysiology of post-seizure Todd's paralysis. Med Sci Monit. 2020 Mar 5;26:e920751.

3 Wong ET, Rude RK, Singer FR, Shaw ST Jr. A high prevalence of hypomagnesemia and hypermagnesemia in hospitalized patients. Am J Clin Pathol. 1983 Mar;79(3):348-52.

\section{Karger's}


4 Soliman HM, Mercan D, Lobo SS, Mélot C, Vincent JL. Development of ionized hypomagnesemia is associated with higher mortality rates. Crit Care Med. 2003 Apr;31(4):1082-7.

5 Tong GM, Rude RK. Magnesium deficiency in critical illness. J Intensive Care Med. 2005 Jan-Feb;20(1):3-17.

6 Haverkamp W, Breithardt G, Camm AJ, Janse MJ, Rosen MR, Antzelevitch C, et al. The potential for QT prolongation and proarrhythmia by non-antiarrhythmic drugs: clinical and regulatory implications. Report on a policy conference of the European Society of Cardiology. Eur Heart J. 2000 Aug;21(15):1216-31.

7 Trinkley KE, Page RL 2nd, Lien H, Yamanouye K, Tisdale JE. QT interval prolongation and the risk of torsades de pointes: essentials for clinicians. Curr Med Res Opin. 2013 Dec;29(12):1719-26. Epub 2013 Sep 23.

8 Krumholz A, Wiebe S, Gronseth G, Shinnar S, Levisohn P, Ting T, et al. Quality standards subcommittee of the American Academy of Neurology; American Epilepsy Society. Practice parameter: evaluating an apparent unprovoked first seizure in adults (an evidence-based review): report of the quality standards subcommittee of the American Academy of Neurology and the American Epilepsy Society. Neurology. 2007 Nov 20;69(21): 1996-2007.

9 Kellinghaus C, Kotagal P. Lateralizing value of Todd's palsy in patients with epilepsy. Neurology. 2004 Jan 27; 62(2):289-91

10 Janett S, Camozzi P, Peeters GG, Lava SA, Simonetti GD, Goeggel Simonetti B, et al. Hypomagnesemia induced by long-term treatment with proton-pump inhibitors. Gastroenterol Res Pract. 2015;2015:951768.

11 Hansen BA, Bruserud $\emptyset$. Hypomagnesemia as a potentially life-threatening adverse effect of omeprazole. Oxf Med Case Reports. 2016 Jul 27;2016(7):147-9.

12 Nijenhuis T, Vallon V, van der Kemp AW, Loffing J, Hoenderop JG, Bindels RJ. Enhanced passive Ca2+ reabsorption and reduced $\mathrm{Mg} 2+$ channel abundance explains thiazide-induced hypocalciuria and hypomagnesemia. J Clin Invest. 2005 Jun;115(6):1651-8.

13 Dai LJ, Friedman PA, Quamme GA. Cellular mechanisms of chlorothiazide and cellular potassium depletion on Mg2+ uptake in mouse distal convoluted tubule cells. Kidney Int. 1997 Apr;51(4):1008-17. 